\title{
Pan-Tilt-Zoom Based Iris Image Capturing System for Unconstrained User Environments at a Distance
}

\author{
Sowon Yoon ${ }^{1}$, Kwanghyuk Bae ${ }^{1}$, Kang Ryoung Park ${ }^{2}$, and Jaihie Kim ${ }^{1}$ \\ ${ }^{1}$ School of Electrical and Electronic Engineering, Yonsei University, \\ Biometrics Engineering Research Center (BERC), Republic of Korea \\ \{swyoon, paero, jhkim\} @yonsei.ac.kr \\ ${ }^{2}$ Division of Digital Media Technology, Sangmyung University, \\ Hongji-Dong, Jongro-Ku, Seoul, Republic of Korea, \\ Biometrics Engineering Research Center (BERC) \\ parkgr@smu.ac.kr
}

\begin{abstract}
This paper proposes a novel iris image capturing system using a pantilt-zoom camera for unconstrained user environments at a distance. To provide wide operating range, we are trying to develop iris image acquisition system using pan-tilt-zoom camera. This paper verifies that iris recognition is possible when iris images are captured with zoom lens at a distance from camera. Iris images are collected by changing the distance from camera to user, zoom factor to maintain face size in an image, and focus lens position. And they are assessed by Hamming distance. Experimental results show that iris images captured at a distance of 1.5$3 \mathrm{~m}$ from camera can be verified as genuine and proposed system has 5-10 cm depth of field. This means that users have freedom of position as $1.5 \mathrm{~m}$ and natural movement of a standing user can be covered.
\end{abstract}

Keywords: Iris image capturing system, pan-tilt-zoom camera, depth of field, depth of focus, operating range.

\section{Introduction}

According to [1], [2], the ideal biometric characteristic has five qualities: robustness, distinctiveness, availability, accessibility, and acceptability. Robustness means unchanging on an individual overtime and repeatable to use. Distinctiveness refers to the existence of wide differences in the pattern among the population. Availability means that the entire population should ideally have this measure in multiples. Accessibility means that features are easily presented to an imaging sensor. Acceptability refers that people do not object to having the measurement taken from them and perceive as non-intrusive.

Iris recognition technology satisfies three of above qualities: robustness, distinctiveness, availability [2], [3], [4], [9]. Robustness is satisfied by the fact that the iris is protected behind eye structures such as eyelid and cornea, and the features of the iris, their placement, size, shape and orientation remain stable and fixed from about one year of age throughout life [3]. Distinctiveness is satisfied by the fact that the iris pattern has 200 or more independent degrees of freedom. It implies that the probability of two different irises agreeing by chance in more than $70 \%$ of their phase sequence is about one in 7 billion [4]. Availability is satisfied by the fact that every 
person has two distinctive irises. These three qualities are recently shown in J. Daugman's paper [9]. In this paper, he probes uniqueness and randomness of the templates for identifying each iris from a database of 632,500 iris images.

Despite these desirable characteristics, iris recognition system is not as popular as other biometric systems such as fingerprint and face. For most of the current iris recognition systems, users try to put their eyes in a certain range because they offer very small operating range [6]. This yields low accessibility and acceptability. Accessibility is low because of two reasons. First, they have low throughput because clear iris images are hard to obtain due to small operating range. Second, the systems require many conditions such as well-trained operator, a cooperative user, adjusted equipment and well-controlled lighting conditions [2]. Acceptability is also low because of three reasons. First, users try to put their eyes in operating range several times, so that capturing in-focus iris images takes much time [5]. Second, once their eyes come into operating range they have to stay still. Third, users keep their eyes close to the camera. These make users uncomfortable. In other words, constraints on position and motion of users in a small operating range drop accessibility for in-focus image acquisition of the iris as well as acceptability for user convenience.

There are several efforts to improve accessibility and acceptability of iris recognition system: Sarnoff Corporation and Mitsubishi Corporation. They all try to improve acceptability by recognizing iris at a distance over $1 \mathrm{~m}$ in common. According to [13], the iris image can be captured at even $10 \mathrm{~m}$ distance between user and camera using a telescope and infrared camera.

Sarnoff Corporation suggests a portal system for iris recognition [6]. Their system takes pictures continuously with high resolution camera while a user passes through the portal at a normal walking pace. This system can capture average 1.5 in-focus iris images. They increase accessibility by high throughput (20 subjects/min) and acceptability by the fact that users just walk through the portal. However, there are three disadvantages of their system. First, their system is not useful for the disabled, children, and tall people because the height of capture volume $(20 \mathrm{~cm}$ width $\times 37 \mathrm{~cm}$ height $\times 10 \mathrm{~cm}$ depth) is small to cover height variation. Second, users should be very cooperative; eyes wide open, look straight ahead, walk at a moderate pace, and try to be recognized. Third, there are many specular reflections in iris images and glasses can corrupt the iris image seriously because 8 illuminators close to the user are used.

Mistubishi Corporation proposes an iris image capturing system using a video camera with wide-field-of-view lens, a high resolution digital still camera with narrow-field-of-view lens and a pan-tilt unit [7]. Wide-field-of-view video camera detects a face and pan-tilt unit rotates to the direction which narrow-field-of-view camera can capture the full face image with high resolution. And then the iris region crops from the face image for recognition. Their system improves acceptability by providing wide operating range using pan-tilt unit and a camera with high resolution and big sized CCD sensor. But three disadvantages remain. Since they use a fixed focal lens for narrow-field-of-view camera, the depth of operating range is limited. And it needs two-camera calibration. Also, users have to stay very still.

This paper proposes a novel iris image acquisition system for unconstrained user environments using a pan-tilt-zoom camera at a distance. To achieve acceptability for user convenience, proposed system offers wide operating range which can cope with height variation and positioning with a camera. Wide width and height of operating 
range are achieved by pan-tilt unit and wide depth is obtained by zoom lens. This system can work with relatively less user cooperation and few equipments. Illuminators do not make few specular reflections in the iris and glasses because two near-infrared illuminators are used at a distance. Thus proposed system does not make serious problem on users who wear glasses.

Overall procedure for capturing the iris image is following. In zoom-out state, user's face is detected. After the face is zoomed in until it fills entire image, high resolution iris image is obtained from the face image. To capture the iris stably in unconstrained user environments, obtaining iris image from full face image is advantageous for catching up the user's movement. If the iris is fully zoomed, small movement at a distance of over $1 \mathrm{~m}$ makes the iris disappeared in the camera view.

In this paper, we introduce overall system configuration and algorithms used. And we probe the feasibility of acquired iris images taken at a distance of $1.5-3 \mathrm{~m}$ between user and camera with adjusting zoom factor to fit the face to whole image. We show accessibility by measuring depth of focus. Because wide depth of focus means that focusing on the iris is easy, as a result, we can get in-focus iris images readily. And we show acceptability that operating range in depth is guaranteed as $1.5 \mathrm{~m}$ and natural movement of a standing user is acceptable because depth of field is $5-10 \mathrm{~cm}$ in whole operating range.

The practical system based on pan-tilt-zoom camera with a light stripe projection is proposed in [14]. This system detects the horizontal position of the user by light stripe projection. It reduces search range of the user's face from $2 \mathrm{D}$ to $1 \mathrm{D}$ and obtains the distance between camera and user's face, so that the initial zoom and focus lens positions are determined. Therefore operating time for searching face, zooming and focusing is greatly reduced.

\section{System Configuration}

Proposed system is designed for providing wide operating range: $1 \mathrm{~m}$ width $\times 1 \mathrm{~m}$ height $\times 1 \mathrm{~m}$ depth at a distance of $1.5-2.5 \mathrm{~m}$. The entire concept of proposed system is shown in Fig. 1. By panning and tilting the width and height of operating range are expanded even if actual field of view of a camera is limited. To cover height variation of about $1 \mathrm{~m}$ at a distance of $1.5 \mathrm{~m}$, tilt range should be $\pm 18.43^{\circ}$. To obtain the iris image robustly allowing user's small movement, zooming into the face instead of the iris is required. While the iris can be missed easily in the state of zooming into the iris, it can remain in the image in the state of zooming into the face under the user's movement. To get iris image with over 150 pixels for its diameter which is medium or high quality [8] from the full face image, the width of CCD camera should be over 1,950 pixels. The depth of operating range is enlarged by zooming. For acquiring iris images with enough size for recognition, according to simple lens equation, required focal lengths at a distance of $1.5 \mathrm{~m}$ and $2.5 \mathrm{~m}$ are $149.87 \mathrm{~mm}$ and $249.78 \mathrm{~mm}$, respectively. Two illuminators of $830 \mathrm{~nm}$ are used here. When illuminated with nearinfrared light in the 700-900 nm band, even dark brown irises reveal rich texture [9].

Fig. 2 shows proposed system configuration: 4 mega-pixel CCD camera, a zoom lens with focal length of 70-300 mm, a pan-tilt unit with pan angle of $360^{\circ}$ and tilt angle of $\pm 45^{\circ}$ and two near-infrared illuminators. To control zoom and focus, stepping motors are used. 


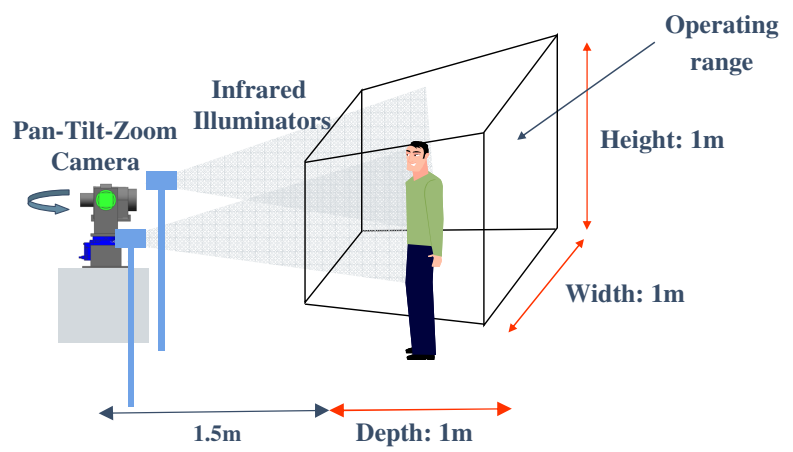

Fig. 1. Concept of proposed system: width and height of operating range are determined by angle of view of camera and angle of pan-tilt unit; depth of operating range is determined by zoom lens

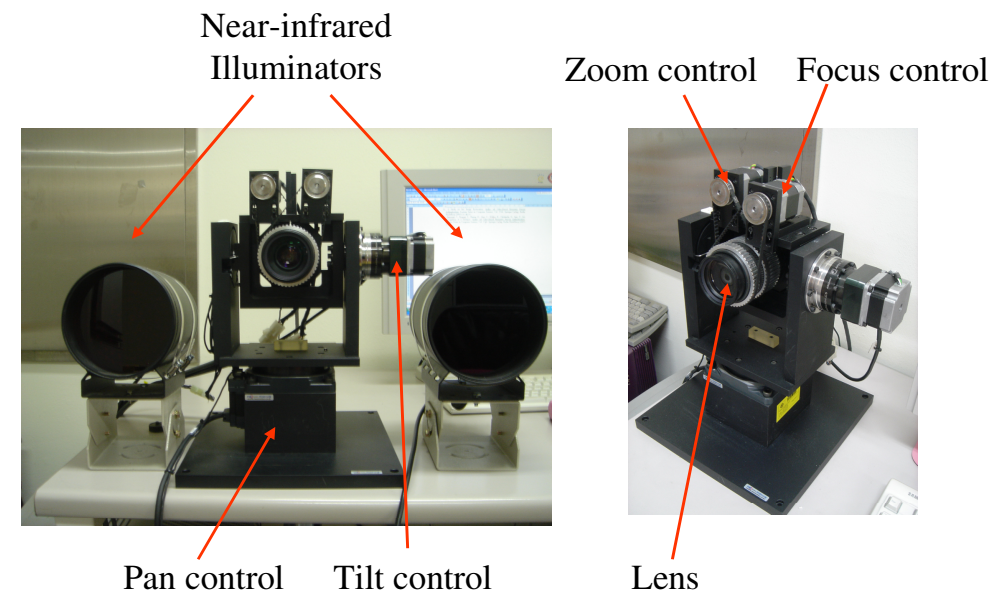

Fig. 2. System configuration: mega-pixel camera, zoom lens, control units for pan, tilt, zoom and focus, and two near-infrared illuminators

The overall procedure for iris image acquisition is following. First, the user's face is detected in zoom-out state using face detector based on AdaBoost algorithm by Viola and Jones [11], which is fast, accurate and robust. Fig. 3(a) shows face detection result in zoom-out state. To be fast and remove unnecessary details which often cause false detection due to high resolution, image size is reduced by $1 / 100$. To evaluate face detection rate of near-infrared images, new database is collected under near-infrared illuminators. It consists of 540 images from 35 persons (19 persons with glasses and 16 persons without glasses). Each person takes pictures at 10 different positions in camera angle shown in Fig. 4 (left). Users with glasses take pictures once 


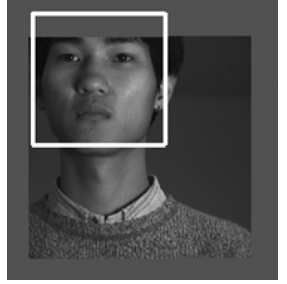

(a)

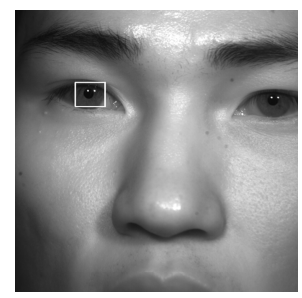

(b)

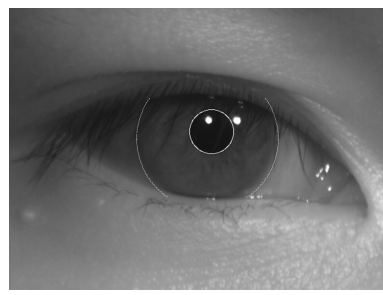

(c)

Fig. 3. Overall procedure for capturing the iris image at a distance. (a) Face detection in wide angle view, (b) iris detection after zooming in the full face, (c) iris localization.

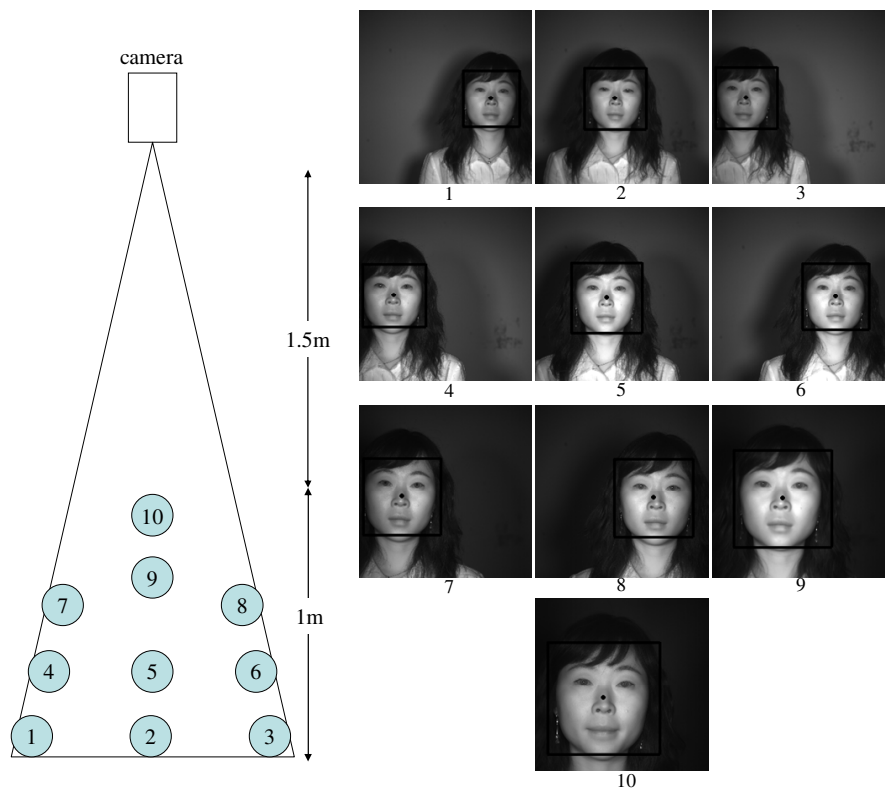

Fig. 4. Positions that face database is collected under near-infrared illuminators (left) and detection results at each position (right). The detection rate in this range is $98.7 \%$.

more without glasses. Fig. 4 (right) shows face detection results at each position. Detection rate is $98.7 \%$. Second, pan and tilt control are performed to place the face in the center of the image. And zoom control to fill the entire image with the user's face is done. Third, iris detection by template matching returns the location and size of the iris. For computational efficiency, search area for template matching is limited on upper half region of the resized image. The result is shown in Fig. 3(b). Fourth, iris localization is required to extract iris pattern. Iris boundary extraction is by circular edge detector [10]. Finally, focus assessments [10], [12] can be performed for capturing in-focus iris image by controlling focus lens movement. 


\section{Experimental Results}

Experiments are designed to probe the feasibility of the iris images captured from proposed system and show accessibility and acceptability of the system by measuring depth of focus, depth of field, and operating range of depth.

In iris recognition, a criterion for image quality of iris can be Hamming distance between two images of same iris: one is acquired from the camera system for iris enrollment, which captures high quality images, and the other is captured from proposed system. If a Hamming distance between enrolled image and the image captured from this system is lower than the threshold, we can say the quality of captured image is good enough to be recognized.

In this paper, the experiment is performed under following two assumptions:

(1) User agrees to iris recognition with cooperation.

(2) User's face appears in zoom-out state.

The procedure of experiment is following. Iris images of user were captured at the distance of every $5 \mathrm{~cm}$ from $140 \mathrm{~cm}$ to $300 \mathrm{~cm}$. In Fig. 5, (a) shows iris image from enrollment system which acquires high quality iris images and (b)-(e) are iris images captured at different distances from the camera of proposed system. At each distance, focus lens position varied from -1000 to 1000 steps. One step moves focus lens position as $1 / 47000$ of full focus range from 1.5 to $\infty$. Fig. 6 shows iris images captured at $2 \mathrm{~m}$ when focus lens position changes. Images at each distance are collected from totally defocused to in-focus. During the experiment, magnification of face was maintained by changing zoom factor according to the distance. And camera parameters such as gain and shutter speed were adjusted to keep the overall intensity of the images manually.

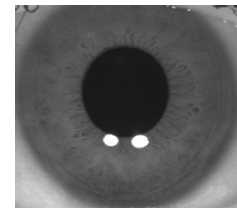

(a) Enrollment

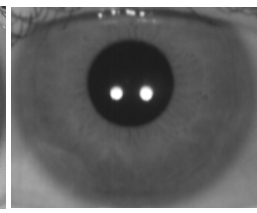

(b) $1.5 \mathrm{~m}$

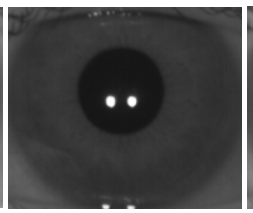

(c) $2.0 \mathrm{~m}$

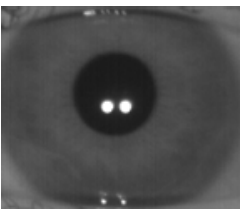

(d) $2.5 \mathrm{~m}$



(e) $3.0 \mathrm{~m}$

Fig. 5. (a) Enrolled image from enrollment device and (b) iris images captured at different distances

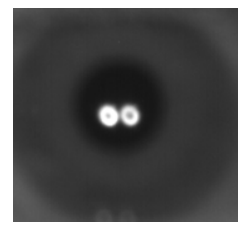

(a) -1000 step

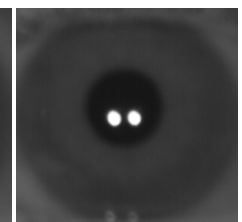

(b) -500 step



(c) 0 step

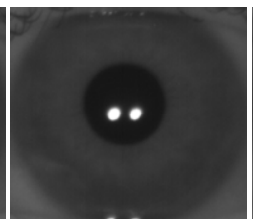

(d) +500 step

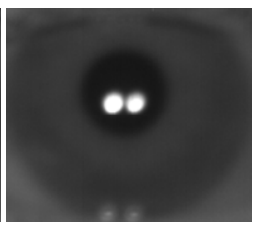

(e) +1000 step

Fig. 6. Iris images captured by changing focus lens position at $2 \mathrm{~m}$ 
Depth of focus is obtained at each distance. Depth of focus is related to the question: 'do we need delicate focusing algorithm?' It can be one of the measurements for accessibility, because wide depth of focus means that focusing on iris is easy.

Depth of field means permissible range for the depth variation of user position at fixed focus lens position. It means acceptability for depth variation. By this, we can prove that proposed system is feasible in a practical situation that a user stands in front of the system. Even if a user tries to stand still for recognition, there exists variation of the position. The variations of width and height are not critical because we capture the full face, thus the iris still remains in the image despite small movement. The variation of depth, however, may be critical because the iris may be out of focus. Depth of field is related to this question: 'is it robust for natural movement of a standing user?'

Depth of operating range is determined by the range that proposed system can capture the iris images which can be recognized. It means acceptability for depth position. Users can stand anywhere in the operating range. We use Gabor wavelet algorithm for creating iris templates which has threshold around 0.32. This threshold is usual in Gabor wavelet [10].

Fig. 7 shows Hamming distance of captured images changing focus lens position at $2 \mathrm{~m}$. As the position of focus lens is changed, defocus images and in-focus images are obtained. Among them, images with lower Hamming distance than threshold can be recognized. The range of focus lens position with lower Hamming distance than threshold is depth of focus.

Depth of focus at each distance is presented in Fig. 8. Range between maximum focus lens position (line with cross) and minimum focus lens position (line with point) means available focus lens position at a certain user distance. Inside that range, depth of field and depth of focus can be measured. At a certain distance, variable range of

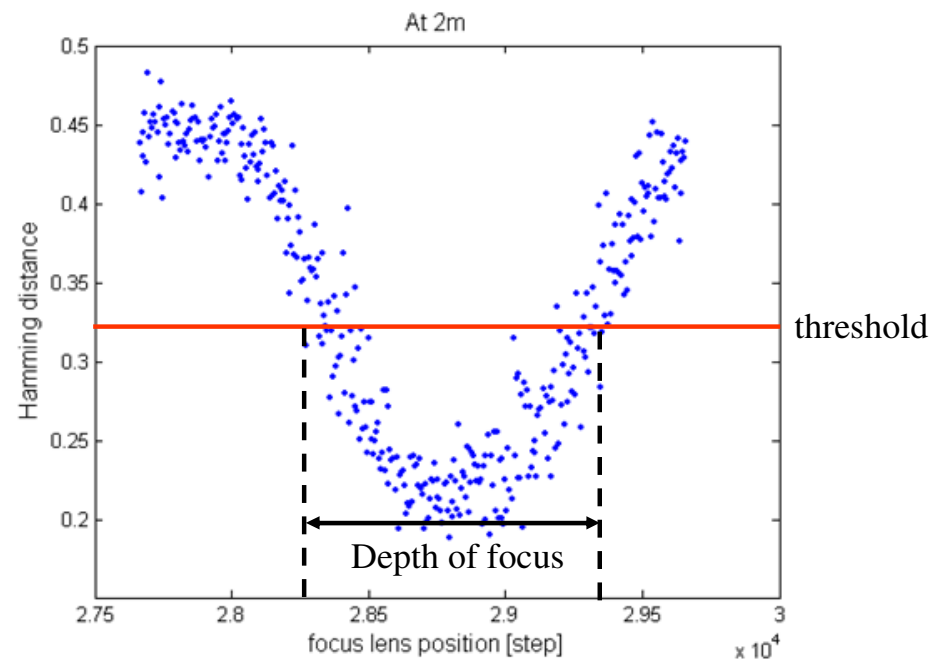

Fig. 7. Hamming distance of iris images captured from proposed system when focus lens position is changed at $2 \mathrm{~m}$ and depth of focus 


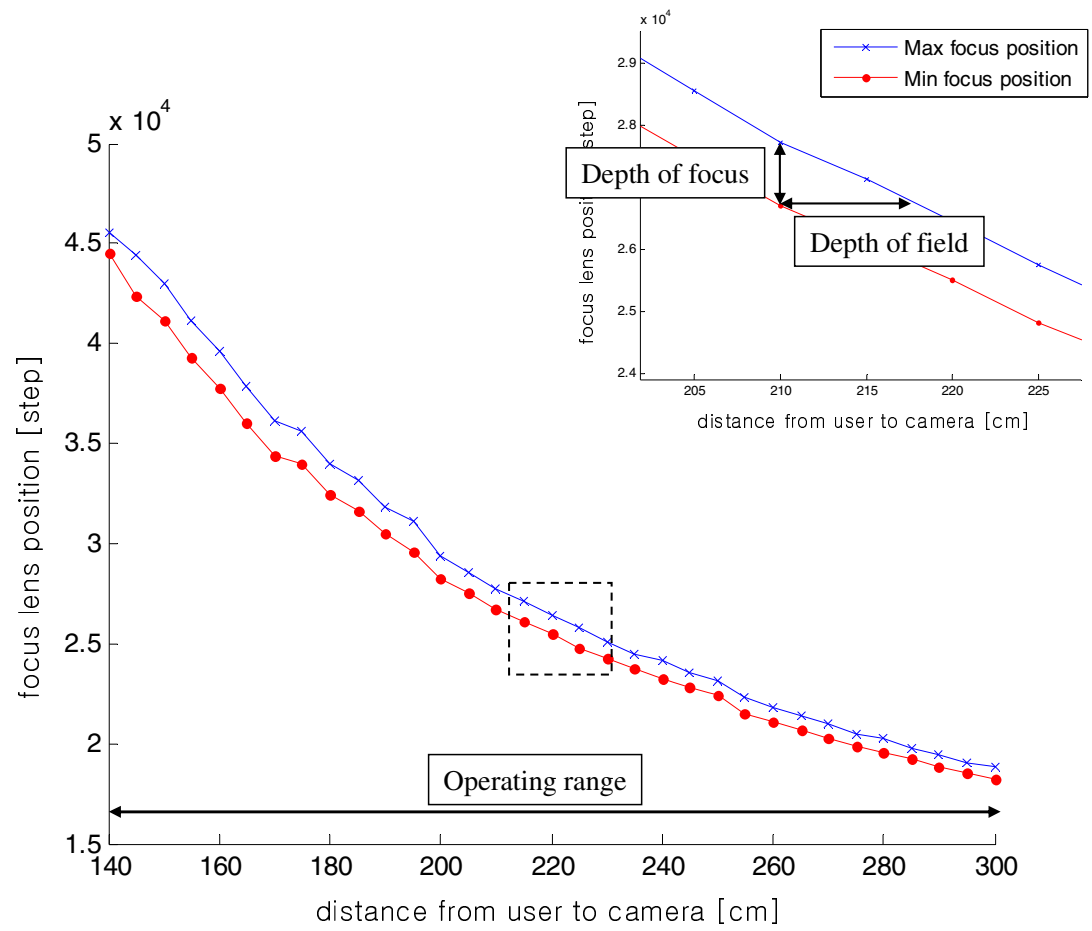

Fig. 8. Depth of focus, depth of field and operating range of proposed system; upper right figure is the enlarged dotted rectangle region of original figure. Line with cross is maximum focus lens position and line with point is minimum focus lens position at each distance.

focus lens is depth of focus. At a certain focus lens position, variable range of distance from user to camera is depth of field. Optimal focus lens position to obtain an in-focus image is changed dependent on the distance. Actual depth of operating range is from 1.4 $\mathrm{m}$ to $3 \mathrm{~m}$, which can cover the target depth range of proposed system.

Fig. 9 shows depth of field and depth of focus at each distance. Depth of field is $4.5-9.5 \mathrm{~cm}$ in whole depth range. This means that depth variation of a standing user which can occur when the user stands naturally can be coped. Thus proposed system can recognize the iris of a user who stands naturally at a distance. Depth of focus varies from 500 steps to 2000 steps. This means that focus lens position within depth of focus from in-focus position can produce recognizable iris images. That is, focus lens control has margin about 1000 steps.

Depth of field increases as distance between user and camera increases. This means that user freedom for movement increases as the distance from camera increases. On the other hand, depth of focus decreases as the distance from camera increases. This means that focusing on the user at a far distance requires accurate and fine searches to find optimal focus lens position. As the distance increases, depth of field increases and we can get high user acceptability. However, focusing is getting difficult because depth of focus decreases. And power of illuminator drops rapidly. Thus we need to adjust camera parameters such as shutter speed and gain automatically. 

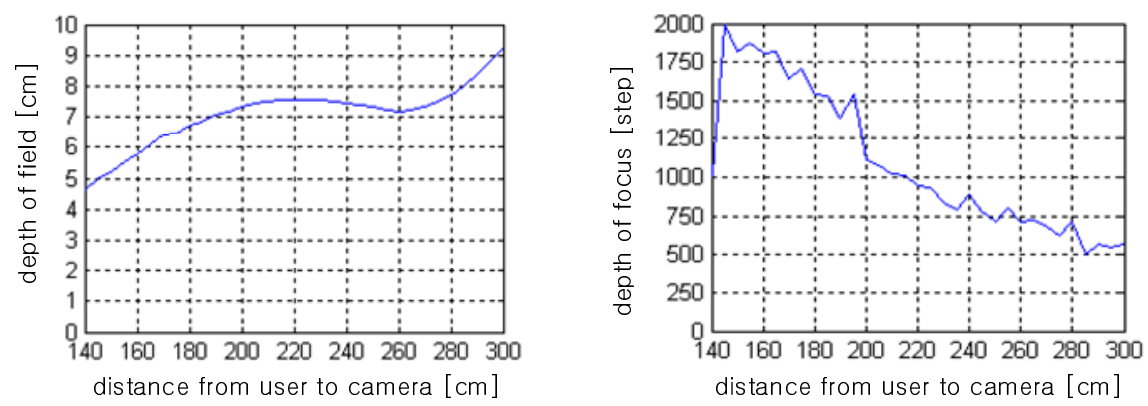

Fig. 9. Depth of field (left) and depth of focus (right) with respect to the distance from user and camera

\section{Conclusions and Future Works}

In this paper, pan-tilt-zoom based iris image acquisition system is proposed. To achieve acceptability for user convenience, this system is designed for wide operating range, $1 \mathrm{~m}$ width $\times 1 \mathrm{~m}$ height $\times 1 \mathrm{~m}$ depth at a distance of 1.5-2.5 m, using pan-tiltzoom camera. To capture iris image stably at a distance of 1.5-2.5 m, zooming into the face size is advantageous because zooming into iris can lose the iris easily by small movement. Face detection, pan-tilt-zoom control and iris localization are performed to get iris image at a distance. Also, we probe the feasibility of acquired iris images at various distances and at various focus lens positions using Hamming distance. Actual depth of operating range is $1.5-3 \mathrm{~m}$, which is larger than the target distance $1.5-2.5 \mathrm{~m}$. Depth of field is $5-9.5 \mathrm{~cm}$, which can cover natural movement of a standing user. That is, proposed system can be used with less user's cooperation and improve acceptability. Depth of focus is 500-2000 steps for whole area. This makes focusing on the iris facilitate and increases accessibility.

Focusing on the iris based on above experimental results will be performed in the future. We still have problems when a user moves rapidly or panning and tilting are fast, because they cause motion blur. And toward more unconstrained environment, pan-tilt-zoom control will be developed by estimating user's movement in more moderate method.

Acknowledgments. This work was supported by the Korea Science and Engineering Foundation (KOSEF) through the Biometrics Engineering Research Center (BERC) at Yonsei University.

\section{References}

1. Wayman, J., Jain, A., Maltoni, D., Maio, D. (eds.): Biometric Systems: Technology, Design and Performance Evaluation. Springer, London (2005)

2. Wayman, J.L.: Fundamentals of Biometric Authentication Technologies. International Journal of Image and Graphics 1(1), 93-113 (2001)

3. Williams, G.O.: Iris Recognition Technology. Aerospace and Electronic Systems Magazine 12(4), 23-29 (1997) 
4. Daugman, J., Downing, C.: Epigenetic Randomness, Complexity and Singularity of Human Iris Patterns. Proceedings of the Royal Society B: Biological Sciences 268(1477), 1737-1740 (2001)

5. Origin, A.: UK Passport Service Biometrics Enrolment Trial. Report (2005), www.passport.gov.uk/downloads/UKPSBiometrics_Enrolment_Trial_Report.pdf

6. Matey, J.R., Naroditsky, O., Hanna, K., Kolczynski, R., Loiacono, D.J., Mangru, S., Tinker, M., Zappia, T.M., Zhao, W.Y.: Iris on the Move: Acquisition of Images for Iris Recognition in Less Constrained Environments. Proceedings of the IEEE 94(11), 19361947 (2006)

7. Guo, G., Jones, M.J., Beardsley, P.: A System for Automatic Iris Capturing. Mitsubishi Electric Research Laboratories, TR2005-044 (2005), http://www.merl.com/publications/ TR2005-044/

8. ANSI INCITS 379-2004.

9. Daugman, J.: Probing the Uniqueness and Randomness of IrisCodes: Results From 200 Billion Iris Pair Comparisons. Proceedings of the IEEE 94(11), 1927-1935 (2006)

10. Daugman, J.: How Iris Recognition Works. IEEE Transactions on Circuits and Systems for Video Technology 14(1), 21-30 (2004)

11. Viola, P., Jones, M.J.: Robust Real-Time Face Detection. International Journal of Computer Vision 57(2), 137-154 (2004)

12. Kang, B.J., Park, K.R.: A Study on Iris Image Restoration. In: Kanade, T., Jain, A., Ratha, N.K. (eds.) AVBPA 2005. LNCS, vol. 3546, pp. 31-40. Springer, Heidelberg (2005)

13. Fancourt, C., Bogoni, L., Hanna, K., Guo, Y., Wildes, R., Takahashi, N., Jain, U.: Iris Recognition at a Distance. In: Kanade, T., Jain, A., Ratha, N.K. (eds.) AVBPA 2005. LNCS, vol. 3546, pp. 1-13. Springer, Heidelberg (2005)

14. Yoon, S., Jung, H.G., Suhr, J.K., Kim, J.: Non-intrusive Iris Image Capturing System Using Light Stripe Projection and Pan-Tilt-Zoom Camera. In: IEEE Computer Society Workshop on Biometrics, accepted for publication (2007) 